\title{
Wideband Monolithic Microwave Integrated Circuit Frequency Converters with GaAs mHEMT Technology
}

\author{
Viktor Krozer, Tom K. Johansen, Torsten Djurhuus, and Jens Vidkjær \\ Technical University of Denmark, Oersted•DTU, Department of Electromagnetic Systems, \\ Oersteds Plads 348, 2800 Kgs. Lyngby, Denmark, Phone:+45-45253769, E-mail:vk@oersted.dtu.dk
}

\begin{abstract}
We present monolithic microwave integrated circuit (MMIC) frequency converter, which can be used for up and down conversion, due to the large RF and IF port bandwidth. The MMIC converters are based on commercially available GaAs mHEMT technology and are comprised of a Gilbert mixer cell core, baluns and combiners. Single ended and balanced configurations $\mathrm{DC}$ and $\mathrm{AC}$ coupled have been investigated. The instantaneous $3 d B$ bandwidth at both the RF and the IF port of the frequency converters is $\sim 20 \mathrm{GHz}$ with excellent amplitude and phase linearity. The predicted conversion gain is around $10 \mathrm{~dB}$. Simulated results are supported by experimental characterization. Good agreement is found between simulations and experiment is found after adjustment of technology parameters.
\end{abstract}

\section{INTRODUCTION}

Ultra-wideband MMICs based on mHEMT technology operating over an instantaneous bandwidth of more $20 \mathrm{GHz}$ are discussed in this paper. The availability of such MMICs reduces front-end complexity and enables reuse of components for different applications in receivers for radar, radiometer and communication applications. One of the key elements in heterodyne receivers is the frequency converter circuit. Double balanced structures based on Gilbert cell mixers have been used earlier and wideband operation has been demonstrated by the authors in SiGe and GaAs technology [1], [2]. Several highly integrated active mixers based on FET technologies have previously been reported [3], [4]. These active mixers have mostly been optimized for down-conversion performance, whereas the active mixers demonstrated here can be used for both upand down-conversion.

This paper focuses on fully integrated DC and AC coupled frequency converters comprised of a Gilbert cell active mixer, balun circuits, balanced buffer amplifiers, and combiners. Aspects of mixer design, such as balance considerations have been discussed in [1], [5]. In this paper we will discuss transistor scaling issues and stability problems. Stability becomes especially important when moving to faster technologies such as e.g. the GaAs mHEMT
D01MH process from OMMIC employed here. We also compare the predicted performance versus experimental results.

\section{DC COUPLED MMIC FREQUENCY CONVERTERS}

The aim of the DC coupled frequency converter was to demonstrate ultra-compact wideband MMIC operation reaching the mm-wave range with competitive performance. In contrast to other circuits, this layout utilizes efficiently the wafer space and is very compact. Fig. 1 shows the layout of the circuit including a Gilbert cell active mixer, two baluns for the LO and RF port, respectively, and a buffer amplifier with source followers and level shifters. All stages are DC coupled and the DC level is adjusted for optimum operation. The circuit is fully differential on input and output. The input matching is achieved by additional $50 \Omega$ resistance together with a short transmission line and buffer amplifiers.

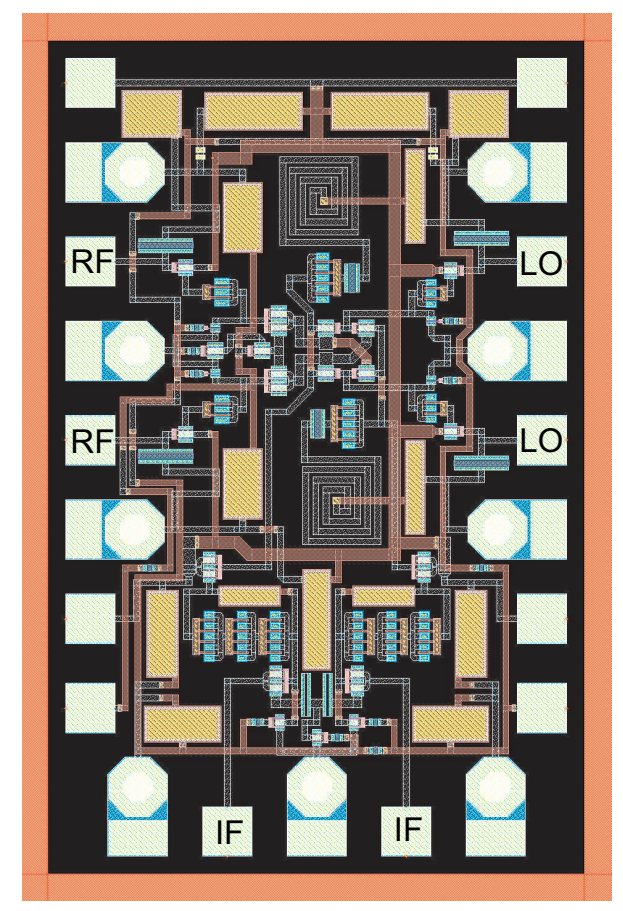

Fig. 1. Layout of an ultra-compact frequency converter. The area is $1 \times 2 \mathrm{~mm}$. 
The basic circuit for the fully differential frequency mixer is shown in fig. 2 together with the buffer circuitry. The RF and LO ports are buffered and can be accessed either differentially or single ended. Especially for the LO port this is convenient if a quadrature oscillator is employed with differential output, which is frequently the case in integrated down/up converters. The output IF port is also differential and is buffered through a buffer amplifier. The buffer amplifier is illustrated in fig 3 .

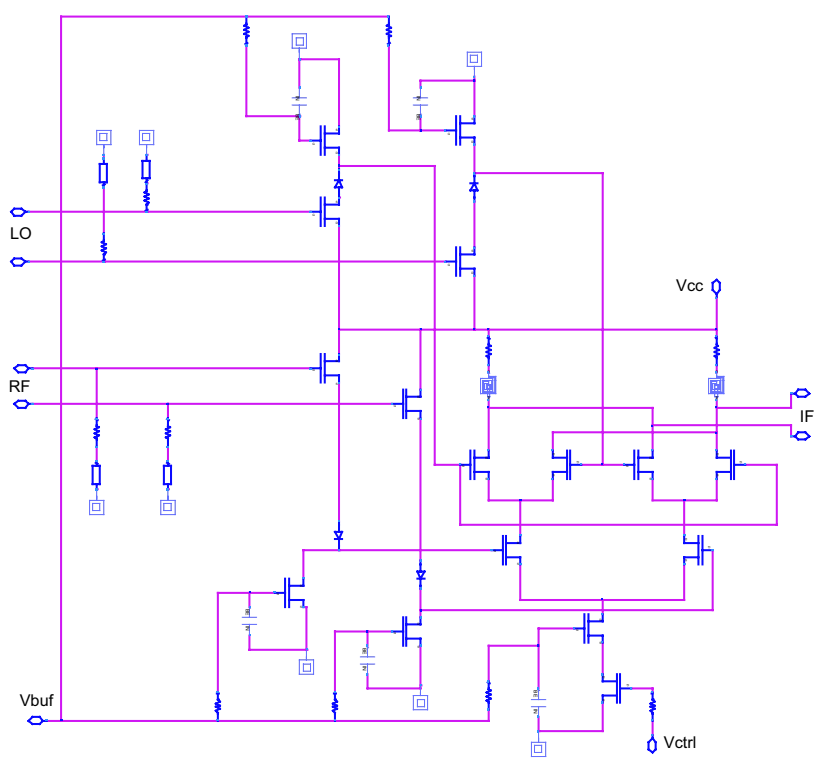

Fig. 2. Simplified circuit of the mixer.

A similar circuit has been used for the baluns, which are integrated on another chip and which occupy roughly quarter of the space of the mixer.

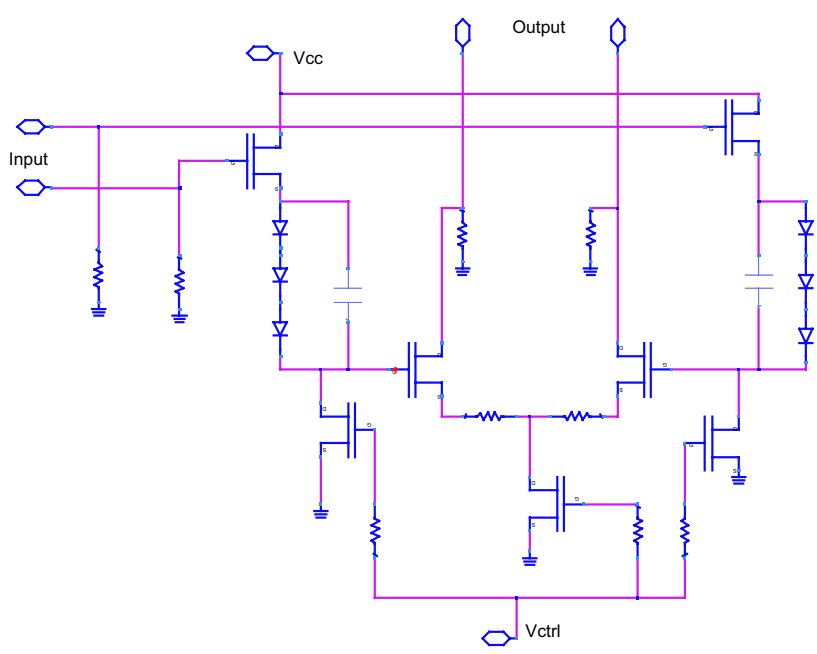

Fig. 3. Simplified circuit of the buffer amplifier integrated with the mixer.

The predicted conversion gain performance versus frequency can be depicted from fig. 4 together with the insertion loss at the RF port. The LO and RF ports are almost identical and well matched up to $\mathrm{mm}$-wave frequencies. Simulations predict a $0.2 \mathrm{~dB}$ bandwidth of $12 \mathrm{GHz}$. However, it can be clearly seen that a resonance exist at around $37 \mathrm{GHz}$. This resonance gives rise to instability problems. Its origin is an interconnect line between the mixer output and the source followers in the buffer circuit. The line length is close to $\lambda / 4$ at this frequency. Eliminating the contribution of the line by reducing
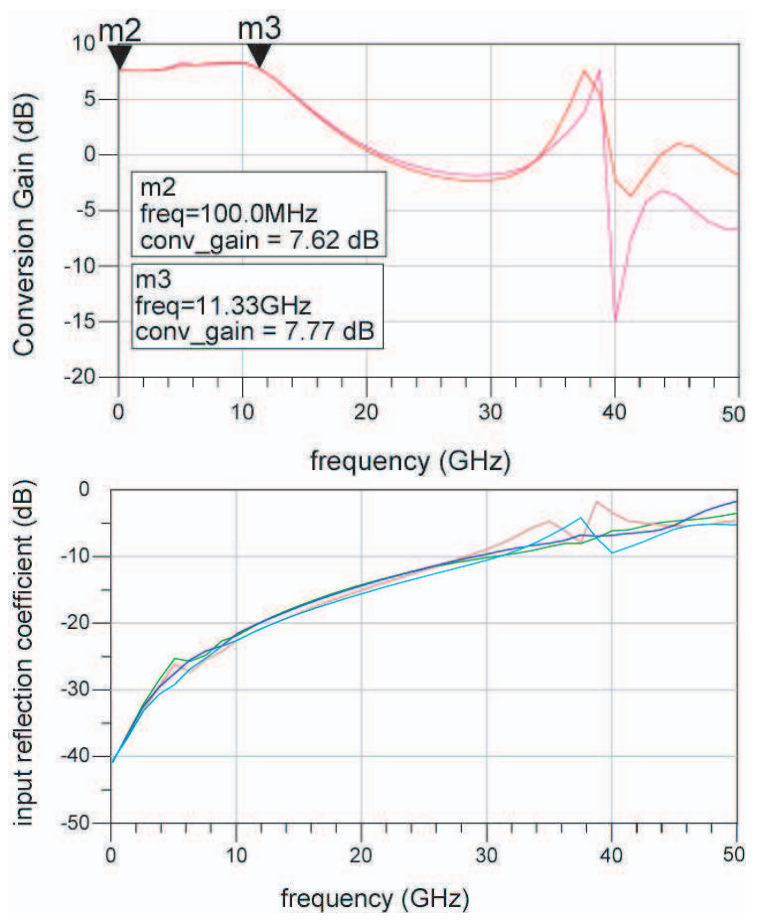

Fig. 4. Simulated conversion gain and insertion loss versus frequency for the frequency converter in fig. 1 .

the line physical length or change in transmission line impedance, the circuit is absolutely stable over the whole frequency range.

The simulated resonance can also be detected in measurements, however due to the losses and a slight shift in the processing parameters the resonance does not give rise to oscillations in measurements.

Measurements have been performed with regards to frequency conversion parameters. The RF port conversion bandwidth was measured by sweeping both the RF and LO signals at a constant IF frequency of $1.2 \mathrm{GHz}$. The IF bandwidth has been measured by keeping the LO signal at a constant frequency and changing the RF signal frequency. The results shown in fig. 5 have been corrected for the cable losses in the measurement system. Both ports exhibit a relatively flat characteristic as a 
function of frequency. Again the circuit works nonoptimally due to non-optimum performance of the current sources. Nevertheless, the operating $3 \mathrm{~dB}$ bandwidth for both ports is close to $15 \mathrm{GHz}$ even in this non-optimum case.
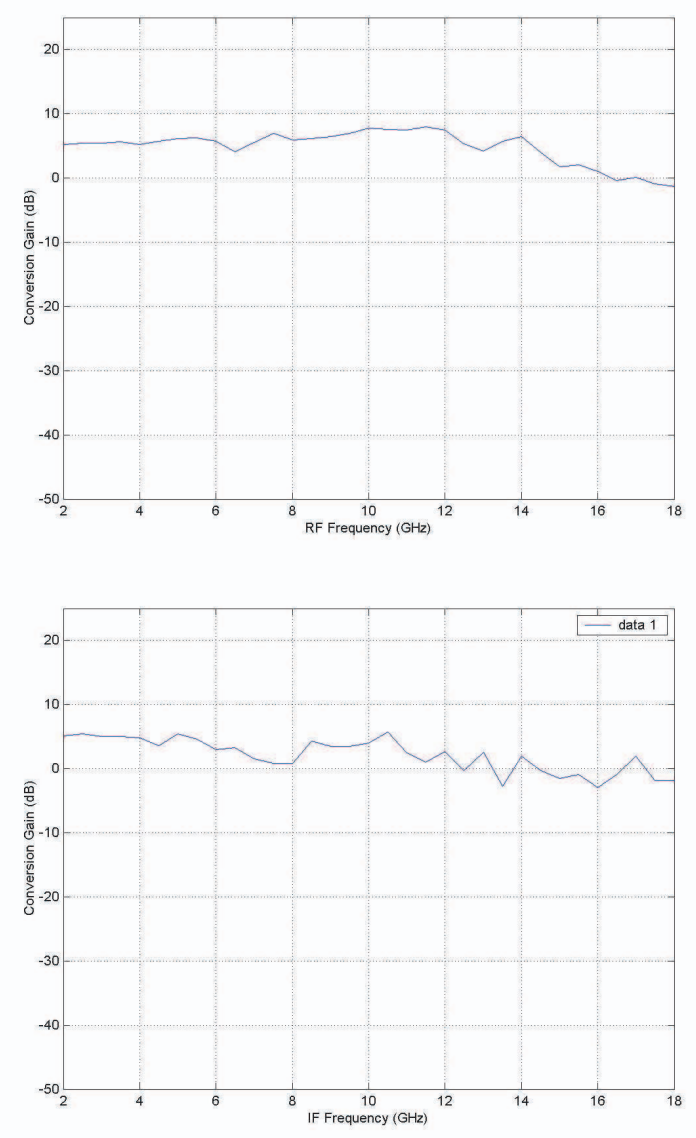

Fig. 5. The measured RF and IF bandwidth versus frequency for the frequency converter in fig. 1 .

One of the major issues in achieving the good results is the proper scaling of the transistors for the transconductance and the switching stage. This point will be discussed in detail in the full paper.

\section{AC COUPLED MMIC FREQUENCY CONVERTERS}

The AC coupled MMIC frequency converter is a modified version of the circuit presented in [1]. It utilizes active baluns based on a common-gate common-source circuit. Input matching in the circuit is achieved with the common-gate FET of the active balun [6]. The GaAs MMIC exhibit slightly wider bandwidth as compared to the SiGe HBT technology. They also exhibit a larger dynamic range. The output of the mixer is DC coupled and inductive peaking is employed for bandwidth enhancement, which is important due to the capacitive loading of the mixer output. The combiner is a modified differential amplifier with wideband performance, level shifter, and source follower stages.

The conversion gain versus frequency is shown in fig. 6. The predicted conversion gain is around $10 \mathrm{~dB}$ and is expected to be flat up to frequencies of $20 \mathrm{GHz}$. The measured values are significantly different. First, the measured conversion gain was determined to be around $-3 \mathrm{~dB}$. Secondly, a strong frequency dependence is visible. Detailed investigation revealed that the common gate mHEMT of the balun exhibited a problem. Taking this into account and including a threshold voltage shift from the nominal $V_{T}=-1.0 \mathrm{~V}$ to $V_{T}=-1.15 \mathrm{~V}$ gave excellent agreement between measured and simulated values. This is verified by the DC bias current characteristic versus the supply voltage as indicated in fig.7

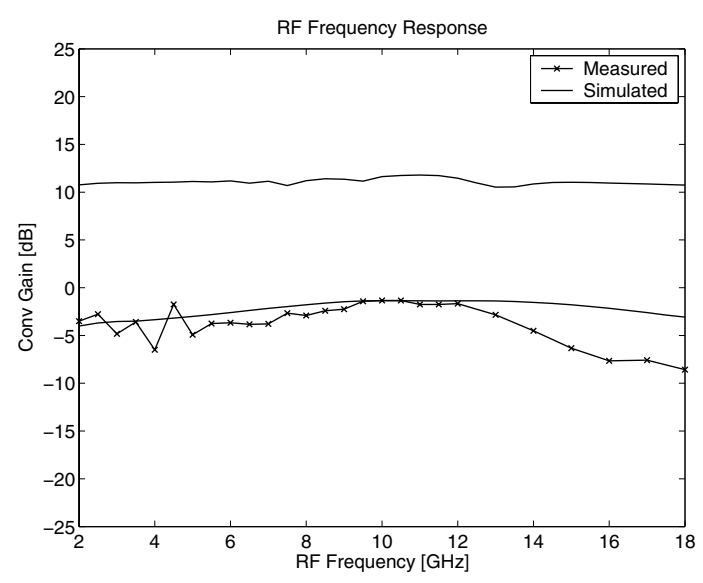

Fig. 6. Conversion gain versus frequency for the MMIC frequency converter.

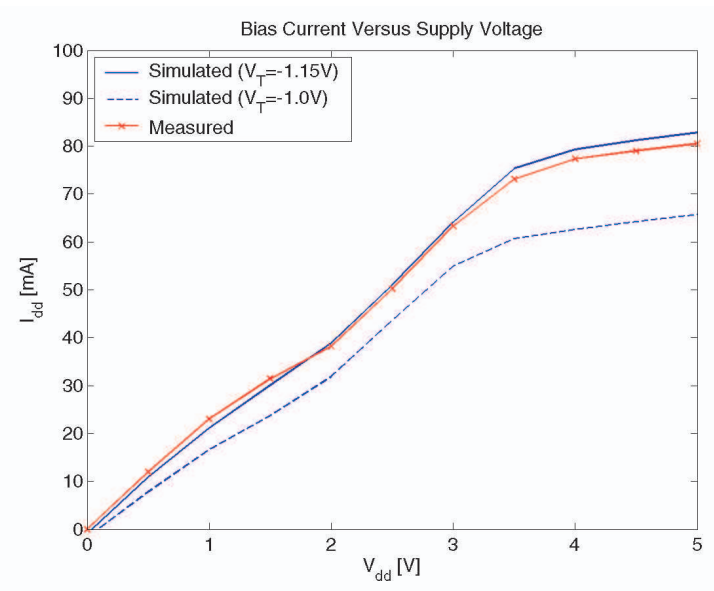

Fig. 7. Conversion gain versus frequency for the MMIC frequency converter.

An interesting feature of this circuit is among oth- 
ers that the noise properties of the converter can be optimized by appropriate choice of the common-gate transistor periphery and bias conditions. Therefore, low-noise operation is feasible with such a circuit. However, the linearity is still a concern and will be further investigated in the future.

\section{CONCLUSIONS}

MMIC circuits with ultra-wideband operation have been designed and fabricated. Results for down/up-converters show a flat conversion gain with a $3 \mathrm{~dB}$ bandwidth in excess of $20 \mathrm{GHz}$. The converter circuits exhibit similar bandwidth at all ports, including the IF port. Therefore, one can employ these circuits as up/down converters with minimum redesign. Very compact circuits have been successfully characterized. Good agreement between simulation and measurement is obtained if the parameters are adjusted for technological spread. Problems with some transistors have been observed in the design, as well as stability problems, due to the large mismatch between the mixer output stage and the source followers. The origin of the instability has been identified and potential stability problems can be alleviated by reducing the interconnect line length between the output of the mixer and the source followers of the buffer amplifier.

The presented circuits demonstrate the feasibility of a compact fully integrated receiver front-end in GaAs technology spanning the frequency range from DC to Ka band. The interesting aspect of this work that the same chip can be used for up-conversion as well as down-conversion.

\section{REFERENCES}

[1] T. K. Johansen, J. Vidkjær, and V. Krozer, "A Highly Integrated GaAs pHEMT Active Mixer for Wideband SAR Systems," in Proc. 2004 Gallium Arsenide and Compound Semiconductor Conf., Amsterdam, The Netherland, 2004, accepted.

[2] V. Krozer, T. K. Johansen, T. Djurhuus, and J. Vidkjær, "Ultra-Wideband Monolithic Microwave Integrated Circuit Solutions in SiGe and GaAs Technology," in Proc. European Microwave Conf., Amsterdam, The Netherland, 2004, accepted.

[3] C.F. Campbell, “"A Wideband pHEMT Downconverter MMIC for Satellite Communication Systems"," in IEEE MTT-S Digest, 1998, pp. 55-58.

[4] C.F. Campbell and J.M. Beall, “'”Design and Performance of a Highly Integrated Wideband Active Downconverter MMIC"," in IEEE Radio Frequency Integrated Circuits Symp., 2001.

[5] T. K. Johansen, V. Krozer, T. Djurhuus, and J. Vidkjær, "Monolithic Microwave Integrated Circuit Solutions for Synthetic Aperture Radar Applications," in Proc. Microwave Technology and Techniques Workshop: Preparing for Future Space Systems, ESA/ESTEC, Noordwijk, The Netherland, 11-12 May 2004.
[6] T. K. Johansen, J. Vidkjær, and V. Krozer, "Ultra-wideband MMICs for remote sensing applications," in Proc. 2003 European Microwave Conference, München, Germany, Oct., 2003. 